\title{
Validation of the Korean Version of the Quality of Life-Cancer Survivors (QOL-CS-K) Questionnaire in Lymphoma Survivors
}

\author{
Juhee Cho, $\mathrm{PhD}^{1,2,3}$ \\ Danbee Kang, $\mathrm{PhD}^{1}$ \\ Im Ryung Kim, RN, MS, AOCN² \\ Won Seog Kim, MD, PhD ${ }^{4}$ \\ Betty Ferrell, RN, PhD ${ }^{5}$ \\ Seok Jin Kim, MD, PhD ${ }^{4,6}$
}

\begin{abstract}
${ }^{1}$ Department of Clinical Research Design and Evaluation, SAIHST, Sungkyunkwan University, Seoul, ${ }^{2}$ Cancer Education Center, Samsung Comprehensive Cancer Center, Samsung Medical Center, Sungkyunkwan University School of Medicine, Seoul, Korea, ${ }^{3}$ Department of Health, Behavior and Society and Epidemiology, Johns Hopkins Bloomberg School of Public Health, Baltimore, MD, USA, ${ }^{4}$ Division of Hematology-Oncology, Department of Medicine, Samsung Medical Center, Sungkyunkwan University School of Medicine, Seoul, Korea, ${ }^{5}$ Nursing Research $\mathcal{E}$ Education, City of Hope Medical Center, Duarte, CA, USA, ${ }^{6}$ Department of Health Sciences and Technology, SAIHST, Sungkyunkwan University, Seoul, Korea
\end{abstract}

\section{Purpose}

The objective of this study was to validate the Korean version of the Quality of Life-Cancer Survivors (QOL-CS-K) in a sample of lymphoma survivors.

\section{Materials and Methods}

We conducted a cross-sectional survey of lymphoma survivors who had survived for at least 24 months since diagnosis. Participants were recruited at the outpatient clinics and at a hospital event in a tertiary hospital in Seoul, Korea. Survivors were asked to complete the QOLCS-K and the European Organization for Research and Treatment of Cancer Quality of Life Core Questionnaire (EORTC QLQ-C30) questionnaires. To determine test-retest reliability, a second questionnaire was sent to participants who completed the first questionnaire adequately. Exploratory factor analysis and Pearson's correlations were used for evaluating reliability and validity of the QOL-CS-K.

\section{Results}

Among 257 survivors, 245 (95.3\%) completed all questionnaires and had no missing data. The mean age of study participants was 52.2 years, $54.9 \%$ were men, and the mean time since diagnosis was 4.0 \pm 1.6 years. The Cronbach's $\alpha$ for the overall QOL-CS-K was 0.90, and the $\alpha$ coefficients for each subscale ranged from 0.73 to 0.83 . The test and retest reliability was 0.88 . Moderate correlations were found between comparable subscales of the QOL-CS-K and subscales of the EORTC QLQ-C30 ( $r=0.51-0.55)$ except for the spiritual well-being subscale of the QOL-CS-K, which did not correlate with any of the EORTC QLQC30 subscales (-0.08 to 0.16).

\section{Conclusion}

The QOL-CS-K is a reliable and valid scale for measuring the QOL in long-term lymphoma survivors.
Correspondence: Seok Jin Kim, MD, PhD Division of Hematology-Oncology, Department of Medicine, Samsung Medical Center, Sungkyunkwan University School of Medicine, 81 Irwon-ro, Gangnam-gu,

Seoul 06351, Korea

Tel: 82-2-3410-1766

Fax: 82-2-3410-1754

E-mail:kstwoh@skku.edu

Received February 20, 2017

Accepted March 17, 2017

Published Online March 30, 2017
Key words

Survival, Quality of life, Lymphoma, Validation studies 


\section{Introduction}

Hodgkin's lymphoma and non-Hodgkin's lymphoma, the most common cancers of the lymphatic system, affect individuals of all ages [1]. Significant advances in the clinical management and treatment of aggressive Hodgkin's lymphoma and non-Hodgkin's lymphoma have improved survival rates, such that they have become chronic diseases for long-term survivors [1]. Given its high incidence and survival rates [2], lymphoma survivorship has been considered an issue in the field of hematology, and a reason for research investigations of the disease's effects on survivors' quality of life (QOL). Most QOL instruments focus on the effects of the diagnosis and initial cancer treatment, whereas the specific concerns and needs of long-term survivors are seldom measured [3].

The European Organization for Research and Treatment of Cancer Quality of Life Core Questionnaire (EORTC QLQC30) [4] and the Functional Assessment of Cancer TherapyAnemia [5] have been the most frequently used instruments for assessing the QOL of lymphoma survivors. However, these measures do not detect new symptoms and concerns that may arise after patients complete their cancer treatments [6]. Some studies have used generic measures, such as the 36-Item Short Form Health Survey [7] to evaluate the QOL of lymphoma survivors [8] as well; however, it also has limitations because it does not address specific problems experienced by cancer survivors [3]. Moreover, although studies have reported that survivors also experience positive changes associated with having cancer, such as post-traumatic growth and appreciation of life [9], most instruments do not assess the positive effects of experience with cancer.

The Quality of Life-Cancer Survivors (QOL-CS) questionnaire is one of the few instruments that was designed specifically for the assessment of QOL in long-term cancer survivors and has been validated or used in different countries [10-12]. The objective of this study was to validate the Korean version of the Quality of Life-Cancer Survivors (QOL-CS-K) in a sample of lymphoma survivors.

\section{Materials and Methods}

\section{Participants}

We conducted a cross-sectional study of consecutive lymphoma survivors who were attending the outpatient hematology/oncology clinic at the Samsung Medical Center in Seoul, Korea, between June 2013 and February 2014. In addition, we recruited survivors who came for a hospital event for the survivorship. The survivors were eligible to participate if they were 18 years of age or older, had been diagnosed with lymphoma more than 24 months before participating in the study were literate in Korean at the time of survey $(n=269)$. Then exclusion criteria were survivors who were diagnosed with multiple cancers or who had psychiatric disorder at the time of the survey.

The first 200 participants who completed the Korean QOLCS-K to re-take it to provide measures to assess its test-retest reliability. The survivors who agreed to re-take the surveys received the survey questions 2 weeks after the first survey with a postage-paid return envelope, and asked to return the completed survey within 2 weeks. The Institutional Review Board of the Samsung Medical Center approved this study, and each participant provided informed written consent.

\section{Measures}

The QOL-CS was developed by researchers of the City of Hope National Medical Center in California, USA, to measure the QOL of long-term cancer survivors [10]. The QOLCS includes 41 items representing the four domains (physical, social, psychological, and spiritual well-being) of cancer-specific QOL [10]. The summed scores for each domain were used as an outcome measure with higher scores indicating a better QOL. To develop the Korean version of the instrument (QOL-CS-K), we obtained copyright permission from Dr. Ferrell, who developed the original QOL-CS. Two bilingual experts translated the QOL-CS to Korean; then, it was back-translated to English by two independent bilingual experts. Based on the experts' opinions of each item, we modified the scale's questions with permission from Dr. Ferrell. After this process, a group of experts, including oncologists, oncology nurses, behavioral scientists, and psychiatrists reviewed and confirmed the instruments' content. In addition, a pilot test of ten survivors confirmed the content validity of the scale. Instead of using the original 11-point Likert scale (0 to 10 ), we used a 4-point Likert scale ranging from 1 (not at all) to 4 (very much) based on the results of the pilot test, expert opinions, and Dr. Ferrell's agreement.

To examine concurrent validity, we used the EORTC QLQC30. This instrument is a 30-item cancer-specific questionnaire, which includes five functioning domains (physical, role, cognitive, emotional, and social), global health and overall health related QOL scales, and several items that assess symptoms commonly reported by patients who have cancer [13]. Participants rated each question on a Likert scale ranging from 1 (not at all) to 4 (very much), excluding global health status, which was rated from 1 (very poor) to 7 (excellent). We scored the EORTC QLQ-C30 items using the scor- 
ing procedures outlined in the manual [14] and linearly transformed the data to yield scores from 0 to 100; a higher score represented a better level of functioning. The EORTC QLQ-C30 has been validated, translated to Korean [15], and has been widely used to measure the QOL of cancer survivors in Korea.

We also collected socio-demographic data, including marital status, education, employment status, and monthly family income. Clinical information, including the stage of cancer at the time of diagnosis and the date of diagnosis, was obtained from the participants' medical records.

\section{Statistical methods}

Data analyses included descriptive statistics (frequencies, means, and standard deviations) and statistics for assessing the questionnaire's psychometric properties. Cronbach's $\alpha$ and the item-total correlation of each subscale of the QOLCS-K were calculated to assess its internal consistency reliability. The stability of the QOL-CS-K was assessed by computing correlations between the participants' initial and retest scores, specifically, the intra-class and Pearson's correlations. In order to measure the questionnaire's concurrent validity, Pearson's correlation coefficients between comparable dimensions of the QOL-CS-K and the EORTC QLQ-C30 were computed. All reported p-values were two-sided and the significance level was set at 0.05 . All analyses were performed using STATA ver. 13 (StataCorp LP, College Station, TX).

\section{Results}

\section{Participants}

Among the 284 eligible survivors, 276 (97.2\%) agreed to participate in the study. Among 276, seven survivors did not return the questionnaires, and 12 had psychological problems at the time of survey. Then final analysis included 257 survivors. The mean age of the study participants was 52.2 years old. Among the participants, 54.9\% were men and 51.8\% were currently employed. The majority of survivors had been diagnosed with non-Hodgkins lymphoma (90.4\%), and the mean time since diagnosis was $4.0 \pm 1.6$ years (Table 1 ).

\section{Feasibility}

Among 257 survivors, 245 completed all questionnaires and had no missing data. There were 12 patients $(4.7 \%)$ who missed at least one item: one patient who missed six items,
Table 1. Participants' socio-demographic and medical characteristics

\begin{tabular}{|c|c|}
\hline Characteristic & No. $(\%)(n=257)$ \\
\hline \multicolumn{2}{|l|}{ Demographics } \\
\hline \multicolumn{2}{|l|}{ Sex } \\
\hline Male & $139(54.9)$ \\
\hline Female & $118(45.9)$ \\
\hline Age, mean \pm SD (yr) & $52.2 \pm 14.0$ \\
\hline \multicolumn{2}{|l|}{ Working status } \\
\hline Housewife & $73(28.6)$ \\
\hline Unemployed & $3(1.2)$ \\
\hline Leave of absence/Loss of job & $47(18.4)$ \\
\hline Employed & $132(51.8)$ \\
\hline \multicolumn{2}{|l|}{ Education } \\
\hline Less than high school & $133(52.2)$ \\
\hline More than College/University & $122(47.8)$ \\
\hline \multicolumn{2}{|l|}{ Marital status } \\
\hline Married & $206(80.5)$ \\
\hline Single/Divorced/Separated/Widowed & $50(15.5)$ \\
\hline \multicolumn{2}{|l|}{ Income $(\$)$} \\
\hline$<4,000$ & $140(55.1)$ \\
\hline$\geq 4,000$ & $114(44.8)$ \\
\hline \multicolumn{2}{|l|}{ Smoking status } \\
\hline Never & $157(61.6)$ \\
\hline Past & $83(32.6)$ \\
\hline Current & $15(5.6)$ \\
\hline \multicolumn{2}{|l|}{ Drinking status } \\
\hline Never & $114(44.5)$ \\
\hline Past & $46(18.0)$ \\
\hline Current & $96(37.5)$ \\
\hline Comorbidity (yes) & $116(45.2)$ \\
\hline Depression, mean \pm SD & $4.6 \pm 4.6$ \\
\hline \multicolumn{2}{|l|}{ ECOG performance status at diagnosis ${ }^{\mathrm{a})}$} \\
\hline $0-1$ & $244(95.3)$ \\
\hline $2+$ & $12(4.7)$ \\
\hline \multicolumn{2}{|l|}{ Clinical characteristics } \\
\hline \multicolumn{2}{|l|}{ Type of diagnosis } \\
\hline Non-Hodgkin's lymphoma & $232(90.3)$ \\
\hline Hodgkin's lymphoma & $25(9.7)$ \\
\hline Time since diagnosis, mean \pm SD (yr) & $4.0 \pm 1.6$ \\
\hline
\end{tabular}

SD, standard deviation; ECOG, Eastern Cooperative Oncology Group. ${ }^{\text {a) }}$ Compared to before the cancer diagnosis.

one patient who missed three items, two patients who missed two items, and eight patients who missed one item. Most frequently missed item was the question asking about menstrual changes or fertility, and four patients (1.6\%) missed the item. Patients who had missing data were significantly older than survivors who completed questionnaire. The mean age of the former was 60.7 , the latter was $51.8(\mathrm{p}=0.03)$. 
Table 2. Internal consistency and item-total correlations on the QOL-CS-K ( $\mathrm{n}=257)$

\begin{tabular}{|c|c|c|c|}
\hline Subscale and item & $\begin{array}{c}\text { Cronbach's alpha } \\
\text { coefficient }\end{array}$ & $\begin{array}{l}\text { Item-total } \\
\text { correlation }\end{array}$ & Mean \pm SD \\
\hline Physical well-being & 0.73 & & $2.5 \pm 0.4$ \\
\hline Fatigue $^{\mathrm{a})}$ & & 0.69 & $2.1 \pm 0.8$ \\
\hline Appetite changes ${ }^{\text {a) }}$ & & 0.68 & $2.7 \pm 0.6$ \\
\hline Aches or pain ${ }^{a)}$ & & 0.64 & $2.4 \pm 0.9$ \\
\hline Sleep changes ${ }^{\text {a) }}$ & & 0.65 & $2.4 \pm 0.9$ \\
\hline Constipation $^{\text {a) }}$ & & 0.51 & $2.7 \pm 0.7$ \\
\hline Nausea $^{a}$ & & 0.49 & $2.8 \pm 0.5$ \\
\hline Menstrual changes or fertility ${ }^{a}$ & & 0.42 & $2.6 \pm 0.8$ \\
\hline Rate your overall physical health & & 0.63 & $2.0 \pm 0.6$ \\
\hline Psychological well-being & 0.82 & & $1.8 \pm 0.4$ \\
\hline Copinga) & & 0.54 & $2.7 \pm 0.6$ \\
\hline Quality of life & & 0.53 & $2.0 \pm 0.6$ \\
\hline Happiness & & 0.31 & $1.5 \pm 1.0$ \\
\hline Control & & 0.29 & $1.5 \pm 0.9$ \\
\hline Satisfying & & 0.35 & $1.6 \pm 1.0$ \\
\hline Concentration/Memory & & 0.29 & $1.3 \pm 0.9$ \\
\hline Usefulness & & 0.03 & $1.7 \pm 1.1$ \\
\hline Altered appearance $\left.{ }^{a}\right)$ & & 0.42 & $2.1 \pm 1.0$ \\
\hline Self-concept $\left.{ }^{\mathrm{a}}\right)$ & & 0.43 & $1.5 \pm 1.0$ \\
\hline Initial-diagnosis distress ${ }^{\text {a) }}$ & & 0.41 & $0.9 \pm 1.0$ \\
\hline Cancer-treatment distress ${ }^{\mathrm{a})}$ & & 0.52 & $1.0 \pm 0.9$ \\
\hline Time of distress since completion of treatment $\left.{ }^{a}\right)$ & & 0.59 & $2.2 \pm 0.8$ \\
\hline Anxiety $^{\text {a) }}$ & & 0.69 & $2.4 \pm 0.8$ \\
\hline Depression $^{\text {a) }}$ & & 0.64 & $2.5 \pm 0.7$ \\
\hline Fear of future diagnostic tests ${ }^{\mathrm{a})}$ & & 0.66 & $2.0 \pm 0.8$ \\
\hline Fear of a second type of cancer $\left.{ }^{a}\right)$ & & 0.73 & $1.6 \pm 0.9$ \\
\hline Fear of a cancer recurrence ${ }^{a)}$ & & 0.74 & $1.5 \pm 1.0$ \\
\hline Fear of cancer spreading (metastasis) ${ }^{\mathrm{a})}$ & & 0.72 & $1.6 \pm 0.8$ \\
\hline Social concerns & 0.83 & & $2.3 \pm 0.5$ \\
\hline Family distress ${ }^{\text {a) }}$ & & 0.74 & $2.2 \pm 0.8$ \\
\hline Support/Others & & 0.31 & $1.9 \pm 1.0$ \\
\hline Personal relationship ${ }^{\text {a) }}$ & & 0.75 & $2.6 \pm 0.7$ \\
\hline Sexualitya) & & 0.60 & $2.4 \pm 0.9$ \\
\hline Employment ${ }^{\text {a) }}$ & & 0.73 & $2.4 \pm 0.9$ \\
\hline Home activity ${ }^{\text {a) }}$ & & 0.78 & $2.4 \pm 0.8$ \\
\hline Feel isolated $^{\mathrm{a})}$ & & 0.79 & $2.6 \pm 0.7$ \\
\hline Financial burden ${ }^{\text {a) }}$ & & 0.69 & $2.2 \pm 0.9$ \\
\hline Spiritual well-being & 0.77 & & $1.5 \pm 0.7$ \\
\hline Important religious activities & & 0.71 & $1.2 \pm 1.2$ \\
\hline Important spiritual activities & & 0.76 & $1.1 \pm 1.1$ \\
\hline Spiritual change & & 0.77 & $1.1 \pm 1.1$ \\
\hline Uncertainty $^{\mathrm{a})}$ & & 0.29 & $2.2 \pm 0.9$ \\
\hline Positive change & & 0.73 & $1.4 \pm 1.0$ \\
\hline Life purpose & & 0.67 & $1.7 \pm 1.0$ \\
\hline Hopefulness & & 0.60 & $1.9 \pm 1.0$ \\
\hline Overall QOL-CS-K ${ }^{\mathrm{b})}$ & 0.90 & & $1.7 \pm 0.4$ \\
\hline
\end{tabular}

QOL-CS-K, Korean version of the Quality of Life-Cancer Survivors questionnaire. ${ }^{\text {a) }}$ This item was reverse scored, b) Cronbach's alpha, mean, and standard deviation (SD) for the entire QOL-CS-K. 
Table 3. Test-retest reliability of the QOL-CS-K

\begin{tabular}{|c|c|c|c|}
\hline \multirow{2}{*}{ Subscale } & \multirow{2}{*}{$\begin{array}{c}\text { No. }^{\text {a) }} \\
(n=179)\end{array}$} & \multicolumn{2}{|c|}{ Correlation coefficients } \\
\hline & & Intra-class & Pearson's \\
\hline Physical & 173 & 0.80 & 0.79 \\
\hline Psychological & 174 & 0.82 & 0.82 \\
\hline Social & 174 & 0.80 & 0.79 \\
\hline Spiritual & 176 & 0.82 & 0.81 \\
\hline Total QOL-CS-K & 164 & 0.88 & 0.87 \\
\hline
\end{tabular}

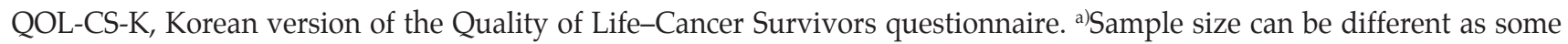
patients missed one to eight items of the questionnaire while they completed other questions.

Table 4. Correlation of the QOL-CS-K with the EORTC QLQ-C30a)

\begin{tabular}{|c|c|c|c|c|c|c|c|c|}
\hline \multirow{3}{*}{ QOL-CS-K subscale } & \multicolumn{8}{|c|}{ EORTC QLQ-C30 } \\
\hline & \multicolumn{2}{|c|}{ General health status } & \multicolumn{2}{|c|}{ Physical functioning } & \multicolumn{2}{|c|}{ Emotional functioning } & \multicolumn{2}{|c|}{ Social functioning } \\
\hline & r-value & p-value & r-value & p-value & r-value & p-value & r-value & p-value \\
\hline Physical & 0.51 & $<0.001$ & 0.53 & $<0.001$ & 0.55 & $<0.001$ & 0.54 & $<0.001$ \\
\hline Psychological & 0.40 & $<0.001$ & 0.35 & $<0.001$ & 0.57 & $<0.001$ & 0.56 & $<0.001$ \\
\hline Social & 0.37 & $<0.001$ & 0.32 & $<0.001$ & 0.46 & $<0.001$ & 0.54 & $<0.001$ \\
\hline Spiritual & 0.16 & 0.008 & -0.08 & 0.211 & 0.07 & 0.275 & 0.04 & 0.573 \\
\hline Total QOL-CS-K & 0.50 & $<0.001$ & 0.38 & $<0.001$ & 0.60 & $<0.001$ & 0.53 & $<0.001$ \\
\hline
\end{tabular}

QOL-CS-K, Korean version of the Quality of Life-Cancer Survivors questionnaire; EORTC QLQ-C30, European Organization

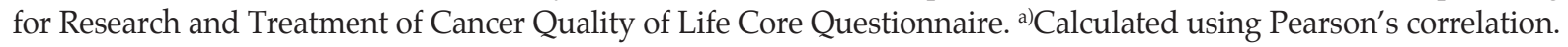

\section{Reliability}

Cronbach's $\alpha$ for the overall QOL-CS-K was 0.90 and the internal consistency for all of the subscales were high (0.730.83) (Table 2). Although the items measuring usefulness (0.03), control (0.29), concentration/memory (0.29), and uncertainty (0.29) had low correlations with their own subscales (e.g., psychological wellbeing), most of the item-to-subscale correlations were moderate to high. For the physical and social well-being subscales, all of the items exhibited higher correlations with their own subscales than with the other subscales. The scores on the subscales are summed so that higher scores represent fewer problems or a better QOL. In our sample, the mean scores for the physical, psychological, social, and spiritual well-being domains were $2.5,1.8,2.3$, and 1.5, respectively.

\section{Test-retest reliability}

Of the 200 participants who agreed to re-take the QOL-CS$\mathrm{K}, 179$ responses were received $(89.5 \%)$. Of the 179 respondents, 164 patients $(89.6 \%)$ completed survey without any missing, and 15 patients missed at least one item ranging from 1 to 8 . Most frequently missed items were items asking about impact of sexual activity $(n=8)$ and menstrual changes or fertility $(n=7)$. The test-retest reliability for the overall QOLCS-K was 0.88 . The physical, psychological, social, and spiritual well-being domains had reliability coefficients of 0.80 , $0.82,0.80$, and 0.82 , respectively (Table 3 ).

\section{Validity}

To examine the concurrent validity of the QOL-CS-K, Pearson's correlation coefficients between the subscales of the EORTC QLQ-C30 and those of the QOL-CS-K were calculated (Table 4). Moderate correlations were found between comparable subscales of the two instruments: (1) the QOL-CS-K physical with the EORTC QLQ-C30 general health status 
$(\mathrm{r}=0.51, \mathrm{p}<0.01)$ and physical functioning $(\mathrm{r}=0.53, \mathrm{p}<0.01)$ subscales; (2) the QOL-CS-K psychological with the EORTC QLQ-C30 emotional health $(\mathrm{r}=0.55, \mathrm{p}<0.01)$ subscale; (3) the QOL-CS-K social with the EORTC QLQ-C30 social functioning $(\mathrm{r}=0.54, \mathrm{p}<0.01)$ subscale; and the (4) QOL-CS-K total score with the score for each subscale of the EORTC QLQ-C30 $(\mathrm{r}=0.38-0.60, \mathrm{p}<0.01)$. However, no significant associations were found between the spiritual well-being subscale of the QOL-CS-K and any of the EORTC QLQ-C30 subscales (-0.08 to 0.16 ).

\section{Discussion}

In this study, the Korean version of the QOL-CS was found to be a reliable and valid measure of QOL among Korean survivors of lymphoma cancer. The four factors that emerged in this analysis are reflected in the four QOL domains: physical, psychological, social, and spiritual domains. The concurrent validity of the QOL-CS-K was supported by their correlations with the EORTC QLQ-C30, except for spiritual wellbeing.

The feasibility of using the QOL-CS-K is good. Of total, 97.5\% of the participants who received the questionnaire responded, and $93.4 \%$ completed all of the questions without any missing. These rates are higher than those of other validation studies [11,12]. The QOL-CS-K seems to be a more feasible instrument because of the organizational changes of the tool we made based on the results of the pilot study and discussions with the original tool developer. We changed the number of response options from the original 11-point Likert scale to a 4-point Likert scale and changed the order of the questions a bit different from the original QOL-CS asking overall QOL, distress, and fear of recurrence first following physical, psychological, and spiritual wellbeing related questions. Several studies have reported differences in responding to survey questions between various racial/ethnic groups. In particular, with Asian populations, the construct validity of the scales tended to improve when there were four rather than 10-point scale or 5-point scale response choices, and we expected our change to a 4-point scale would enhance our tool's feasibility [16]. Therefore, it is important to consider whether response choices are culturally appropriate as well as the translated questionnaires when a tool is adapted and validated in different languages.

The study's results revealed that the physical, psychological, social, and spiritual subscales of the QOL-CS-K had good psychometric properties. In addition, we found strong to moderate correlations between the individual items and their subscales, except for three items pertaining to control, concentration/memory, and usefulness. However, these items also had weak correlations with the subscales in the original study [10] and in a study of the Dutch version of the QOL-CS with survivors of prostate cancer [12]. More studies are necessary for further validation of the QOL-CS-K with different cancer survivors.

In our study, the lymphoma survivors seemed to have a relatively good QOL compared to the healthy comparison groups reported in previous studies [17]. However, many of the survivors reported persistently low psychological and spiritual well-being related to fear of the cancer's recurrence, insufficient support, and a lack of purpose in life and hope. This result might be due to the possibility that physical symptoms were closely followed up and cared for by oncologists during the surveillance period, whereas psychological and/or spiritual well-being issues were rarely discussed with the survivors, which worsened their psychological problems, such as depression and anxiety disorder $[18,19]$. In recent years, spiritual wellbeing has been considered as a core aspect of QOL in the cancer survivors as it includes critical components of the survivorship trajectory, such as finding a purpose and meaning in life. Yet, there is limited evidence with lymphoma survivors because most of the QOL studies with these survivors use tools, such as the EORTC QLQ-C30 or the SF-36, which do not assess the spiritual wellbeing of the survivors. Studies with larger and representative samples that use the QOL-CS-K are necessary to provide a more comprehensive assessment of the QOL of long-term survivors of lymphoma.

Regarding the concurrent validity of the QOL-CS-K, there were moderate correlations between the physical, psychological, and social-related subscales of the QOL-CS-K and the EORTC QLQ-C30, and all of them were statistically significant. However, there was no correlation between the spiritual well-being subscale of the QOL-CS-K and the physical, emotional, or social functioning subscales of the EORTC QLQ-C30, suggesting that the spiritual well-being subscale of the QOL-CS-K measures unique aspects of QOL among lymphoma survivors, which are not assessed by the EORTC QLQ-C30.

This study has several limitations. First, in an effort to minimize respondents' burden, the concurrent validity of the spiritual well-being subscale of the QOL-CS-K was not examined using separate instruments, such as the Faith, Importance and Influence, Community, and Address (FICA) Spiritual Assessment Tool [20], the Mental Adjustment to Cancer (MAC) Scale [21], or the Spiritual Needs Scale for Korean Patients with Cancer [22]. However, in a study with a Japanese participants, who have a similar cultural background with Koreans, the spiritual well-being domain of the Japanese version of the QOL-CS was strongly correlated with spiritual well-being as measured by the MAC subscale [11], 
indicating possible concurrent validity of the spiritual wellbeing domain of the QOL-CS-K. Second, as the instrument was validated based on a sample of Korean lymphoma survivors, it may not be able applicable to other cancer survivors with different needs and issues. Nevertheless, we found similar reliability and validity results on the physical, psychological, social, and spiritual well-being subscales with other validity studies [12]. The Dutch and Japanese versions of the QOL-CS questionnaire were validated with prostate and lung cancer patients, respectively, and they have been widely used with survivors of other cancers [23], suggesting that the QOL-CS-K might also be feasible for use with survivors of other cancers. Third, it is possible that response bias among those who were willing to answer the questionnaire twice might have confounded the results, as second-time respondents might have differences from first-time respondents. However, the test-retest reliability was good for all the subgroups of patients.

In conclusion, our study confirmed that the QOL-CS-K (Supplementary Material) is a reliable and valid scale for measuring lymphoma survivors' QOL. It can be used to assess the QOL of long-term as well as intermediate cancer survivors, and measure QOL aspects that affect survivors' daily lives, including physical, psychosocial, and spiritual aspects. Although there is a need to refine the QOL-CS-K, the current instrument should help oncologists assess difficulties experienced by lymphoma survivors and provide more patient-centered care to improve their QOL and health outcomes.

\section{Electronic Supplementary Material}

Supplementary materials are available at Cancer Research and Treatment website (http:// www.e-crt.org).

\section{Conflicts of Interest}

Conflict of interest relevant to this article was not reported.

\section{Acknowledgments}

This study was supported by a grant from the 20-20 project of Samsung Medical Center.

\section{References}

1. Park HJ, Park EH, Jung KW, Kong HJ, Won YJ, Lee JY, et al. Statistics of hematologic malignancies in Korea: incidence, prevalence and survival rates from 1999 to 2008. Korean J Hematol. 2012;47:28-38.

2. Hewitt M, Greenfield S, Stovall E. From cancer patient to cancer survivor: lost in transition. Washington, DC: The National Academies Press; 2005.

3. Arden-Close E, Pacey A, Eiser C. Health-related quality of life in survivors of lymphoma: a systematic review and methodological critique. Leuk Lymphoma. 2010;51:628-40.

4. Donnelly S. Quality-of-life assessment in advanced cancer. Curr Oncol Rep. 2000;2:338-42.

5. Cella D. The Functional Assessment of Cancer Therapy-Anemia (FACT-An) Scale: a new tool for the assessment of outcomes in cancer anemia and fatigue. Semin Hematol. 1997;34 (3 Suppl 2):13-9.

6. Escobar A, Trujillo-Martin Mdel M, Rueda A, Perez-Ruiz E, Avis NE, Bilbao A. Cross-cultural adaptation, reliability and validity of the Spanish version of the Quality of Life in Adult Cancer Survivors (QLACS) questionnaire: application in a sample of short-term survivors. Health Qual Life Outcomes. 2015;13:182.

7. Ware JE Jr, Snow KK, Kosinski M, Gandek B. SF-36 health survey: manual and interpretation guide. Boston, MA: The Health Institute, New England Medical Center; 1993.
8. Smith SK, Mayer DK, Zimmerman S, Williams CS, Benecha H, Ganz PA, et al. Quality of life among long-term survivors of non-Hodgkin lymphoma: a follow-up study. J Clin Oncol. 2013;31:272-9.

9. Jeon M, Yoo IY, Kim S, Lee J. Post-traumatic growth in survivors of allogeneic hematopoietic stem cell transplantation. Psychooncology. 2015;24:871-7.

10. Ferrell BR, Dow KH, Grant M. Measurement of the quality of life in cancer survivors. Qual Life Res. 1995;4:523-31.

11. Fujimori M, Kobayakawa M, Nakaya N, Nagai K, Nishiwaki $\mathrm{Y}$, Inagaki M, et al. Psychometric properties of the Japanese version of the Quality Of Life-Cancer Survivors Instrument. Qual Life Res. 2006;15:1633-8.

12. van Dis FW, Mols F, Vingerhoets AJ, Ferrell B, van de PollFranse LV. A validation study of the Dutch version of the Quality of Life-Cancer Survivor (QOL-CS) questionnaire in a group of prostate cancer survivors. Qual Life Res. 2006;15: 1607-12.

13. Aaronson NK, Ahmedzai S, Bergman B, Bullinger M, Cull A, Duez NJ, et al. The European Organization for Research and Treatment of Cancer QLQ-C30: a quality-of-life instrument for use in international clinical trials in oncology. J Natl Cancer Inst. 1993;85:365-76.

14. Fayers PM, Aaronson NK, Bjordal K, Groenvold M, Curran D, Bottomley A. The EORTC QLQ-C30 scoring manual. 3rd ed. 
Brussels: European Organisation for Research and Treatment of Cancer; 2001.

15. Yun YH, Park YS, Lee ES, Bang SM, Heo DS, Park SY, et al. Validation of the Korean version of the EORTC QLQ-C30. Qual Life Res. 2004;13:863-8.

16. Lee JW, Jones PS, Mineyama Y, Zhang XE. Cultural differences in responses to a Likert scale. Res Nurs Health. 2002;25: 295-306.

17. Costanzo ES, Ryff CD, Singer BH. Psychosocial adjustment among cancer survivors: findings from a national survey of health and well-being. Health Psychol. 2009;28:147-56.

18. Koenig HG. Research on religion, spirituality, and mental health: a review. Can J Psychiatry. 2009;54:283-91.

19. Gonzalez P, Castaneda SF, Dale J, Medeiros EA, Buelna C, Nunez A, et al. Spiritual well-being and depressive symptoms among cancer survivors. Support Care Cancer. 2014;22: 2393-400.

20. Borneman T, Ferrell B, Puchalski CM. Evaluation of the FICA tool for spiritual assessment. J Pain Symptom Manage. 2010; 40:163-73.

21. Watson M, Greer S, Young J, Inayat Q, Burgess C, Robertson B. Development of a questionnaire measure of adjustment to cancer: the MAC scale. Psychol Med. 1988;18:203-9.

22. Yong J, Kim J, Han SS, Puchalski CM. Development and validation of a scale assessing spiritual needs for Korean patients with cancer. J Palliat Care. 2008;24:240-6.

23. Allart-Vorelli P, Porro B, Baguet F, Michel A, Cousson-Gelie F. Haematological cancer and quality of life: a systematic literature review. Blood Cancer J. 2015;5:e305. 